\title{
An Automatic Test Equipment for Automotive Engine Ignitors
}

\author{
KENZO WATANABE, MEMBER, IEEE, AND MASAYUKI MOCHIZUKI
}

\begin{abstract}
Equipment has been developed for testing automotive engine ignitors automatically. It consists of a microcomputer, engine simulator, and measurement unit. Given the ignitor specifications to be tested, the microcomputer calculates the engine state and sets it into the engine simulator. The simulator then produces the signals necessary to operate the ignitor. The advance angle and the dwell time of the ignitor are measured by the measurement unit with the accuracy of $0.1^{\circ}$ in crank angle and of $0.1 \mathrm{~ms}$, respectively. This process is repeated until all the test items are covered. Time required for the test is only a few seconds. The result is plotted, if desired, as a function of engine speed and load.
\end{abstract}

\section{INTRODUCTION}

$\mathrm{F}^{2}$ OR THE LAST 15 years, automotive electronics has made great progress together with semiconductor technology. The motivation was the social need of protecting an environment against exhaust pollutants and of saving energy resources. Therefore, emphasis has been placed on the electronic engine control. The first such system was a fully transistorized ignitor [1]. The high spark energy produced by this ignitor has made it possible to ignite a lean fuel mixture, and thereby to reduce pollutants as well as the fuel consumption. Following the high-energy ignitor, the electronic advance systems such as Lean Burn of Chrysler [2], EEC of Ford [3], and MISAR of GM [4] have been developed to control the spark timing as a function of engine speed, load (manifold pressure), temperature, and recirculated rate of exhaust gas.

There is no doubt that these innovative systems have, in combination with the recent fuel-metering systems, greatly improved the performance of modern vehicles [5]. Their faults are, however, fatal to automobiles, and thus they pose a new engineering problem of quality management, diagnosis, and maintenance [6]. To solve this problem, automatic test equipment (ATE) has been developed which allows quick characterization of engine ignitors. A few systems have been already proposed to this aim [7], [8], but they are not versatile because they are hardware-oriented systems. In the present ATE, all that is required for an operator to do is to input the specifications of the ignitor to be tested. The ATE then proceeds to measure the spark timing and the dwell time. This paper describes its construction and the principles of operation. Test examples are also presented.

Manuscript received March 5, 1984.

K. Watanabe is with the Research Institute of Electronics, Shizuoka University, Hamamatsu 432, Japan.

M. Mochizuki was with the Research Institute of Electronics, Shizuoka University, Hamamatsu 432, Japan. He is now with Murata Machinery Corporation Ltd., Inuyama 484, Japan.

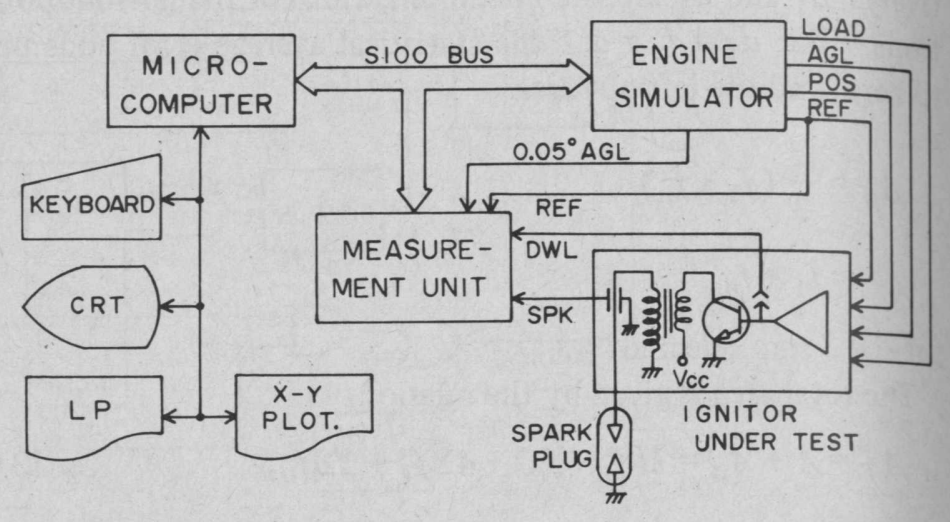

Fig. 1. The block diagram of the automatic test equipment for automotive engine ignitors.

\section{SySTEM DESCRIPTION}

\section{A. Hardware Architecture}

The block diagram of the ATE is shown in Fig. 1. It consists of the microcomputer unit, the engine simulator, and the measurement unit. The test is accomplished as follows. First, an operator specifies the input signals necessary to operate the ignitor under test and the ranges of engine speed and load (manifold pressure) to the microcomputer through a keyboard. Based on these specifications, the microcomputer calculates the first engine state and sends it to the engine simulator. The simulator then generates the signals and operates the ignitor. The generated signals include the reference (REF), position (POS), and angle (AGL) signals, and the load (LOAD) signal in analog form. The REF signal indexes the top-dead-center (TDC) of an engine piston. The POS signals simulate the crankshaft position sensors. Two AGL signals are produced; one is $0.05^{\circ}$ AGL whose period corresponds to $0.05^{\circ}$ in crank angle (CA). The other is the AGL whose period can be changed so that it corresponds to 0.5 , 1,2 , or $5^{\circ}$ in CA. The choice is made by the ignitor specifications. These signals can cover almost all the ignitors now available. Pulse transformers are also incorporated into the simulator to simulate the signals from the magnetic crankshaft position sensors.

The measurement unit measures the advance angle and the dwell time of the ignitor while monitoring the operation of the engine simulator. For this, it receives two signals from the ignitor; one is the dwell (DWL) signal obtainable at the base terminal of an ignitor transistor, and the other is the spark (SPK) signal obtained by the capacitive probe coupled to a spark-plug wire. Because the SPK signal is heavily atten- 


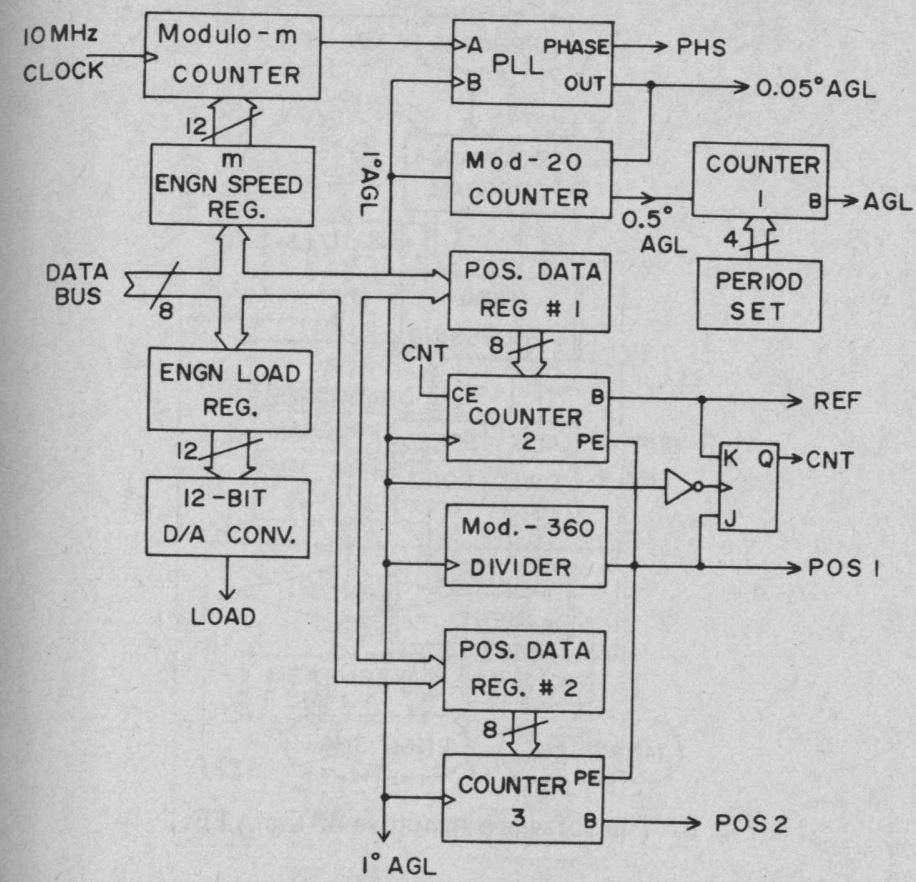

Fig. 2. The schematic diagram of the engine simulator.

uated by the probe, this method of obtaining the SPK signal also allows the measurement of the spark energy which would otherwise destroy semiconductor devices involved in the unit. The accuracy in advance and dwell measurement is specified to be $0.1^{\circ} \mathrm{CA}$ and $0.1 \mathrm{~ms}$, respectively. When the measurement is completed, the microcomputer is instructed to store the data and then set the next engine state. This process is repeated until all the test items are covered. Then, the microcomputer waits for the output command for one device; a CRT, an $X-Y$ plotter, or a line printer, the result should be displayed. The detailed description of each unit will be given next.

1) Microcomputer Unit: A commercially available microcomputer (CPU) is used for the unit. The CPU is a Z- 80 operating at 4-MHz clock. It communicates with external devices through the S-100 bus which includes eight input/output ports.

2) Engine Simulator: The function of this unit is to generate the engine speed, load, and crankshaft position signals to drive the ignitor under test. These signals should be compatible with almost all the ignitors now available. Thus referring to presently available ignitor specifications, the engine speed ranges from 600 to $12000 \mathrm{rpm}$ and the resolution of $100 \mathrm{rpm}$ are specified to the unit. In each engine state, it should also produce the $0.05^{\circ} \mathrm{AGL}$ signal in order to measure the advance angle with $0.1^{\circ}$-CA accuracy. The block diagram of the engine simulator, developed by reflecting these requirements on the hardware algorithm, is shown in Fig. 2. The key in this diagram is the rpm-to-frequency converter consisting of the programmable modulo- $m$ counter, phase-lock-loop (PLL), and modulo-20 counter. The engine speed $N(\mathrm{rpm})$ is related to the frequencies $f_{r}, f_{d}$, and $f_{m}$ of the REF, $1^{\circ} \mathrm{AGL}$, and $0.05^{\circ}$. AGL signals, respectively, as

$$
N=60 f_{r}=f_{d} / 6=f_{m} / 120 .
$$

Thus for $600 \leqslant N \leqslant 12000, f_{m}$ ranges from $72 \mathrm{kHz}$ to 1.44 $\mathrm{MHz}$. Let us assume here to generate $0.05^{\circ}$-AGL signal by

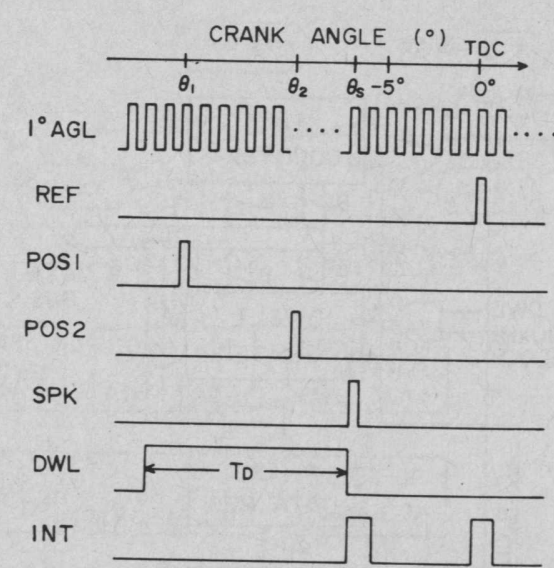

Fig. 3. Timing sequence of the engine simulation signals and the measurement.

dividing a clock signal. In order to attain 100-rpm resolution at $12000 \mathrm{rpm}$, the divider ratio should be larger than 120 . The clock frequency then should be higher than $172.8 \mathrm{MHz}$, which is intolerable to conventional TTL and CMOS counters. This difficulty is overcome in Fig. 2 by combining division and multiplication. Dividing $10-\mathrm{MHz}$ clock signal, the modulo-m counter produces the $1^{\circ}$-AGL signal. The ratio $m$ programmed by the microcomputer then takes an integer in the range from 139 to 2778 , depending on the engine speed. Since $m$ is larger than 120 , the resolution of $100 \mathrm{rpm}$ is assured. The PLL multiplies, in combination with the modulo20 counter in the feedback path, the $1^{\circ}$-AGL signal by 20 to produce the $0.05^{\circ}$-AGL signal. It also produces the phase (PHS) signal whose width is modulated by the phase difference between the $1^{\circ}$-AGL signals at ports $A$ and $B$. This signal is sent to the measurement unit to monitor the operation of the rpm-to-frequency converter.

Once the $1^{\circ}$-AGL signal is obtained, the REF and POS signals can be generated straightforwardly. Their timing is shown in Fig. 3. The POS1 signal preceding the POS2 and REF signals is obtained by dividing the $1^{\circ}$-AGL signal using the modulo-360 counter. The POS2 and REF signals can be obtained then by delaying the POS1 signal using programmable counters. The crank angle $\theta_{1}$ between the POS1 and REF signals and that between the POS1 and POS2 signals, $\theta_{1}-\theta_{2}$, are set into the position data registers 1 and 2 , respectively, based on the ignitor specifications. The simulator shown in Fig. 2 assumes the ignitor having three crankshaft position sensors, but it can be easily extended to more complicated ignitors by incorporating the paired position data registers and programmable counters. Besides the position signals, some recent ignitors require the angle signals. To be compatible with such ignitors, the simulator incorporates the counter 1 in Fig. 2 which produces the AGL signal by dividing the $0.5^{\circ}$-AGL available from the modulo- 20 counter. Depending on the ignitor, its period is set manually into the 4-bit register.

The 12-bit A/D converter plays the roll of an air-flow meter or a manifold pressure sensor. It produces the LOAD signal appropriate for the ignitor by converting the load data inputted through a keyboard into an analog voltage.

3) Measurement Unit: The schematic diagram of the unit is shown in Fig. 4. Its function is to measure the advance angle and the dwell time of the ignitor while monitoring the 


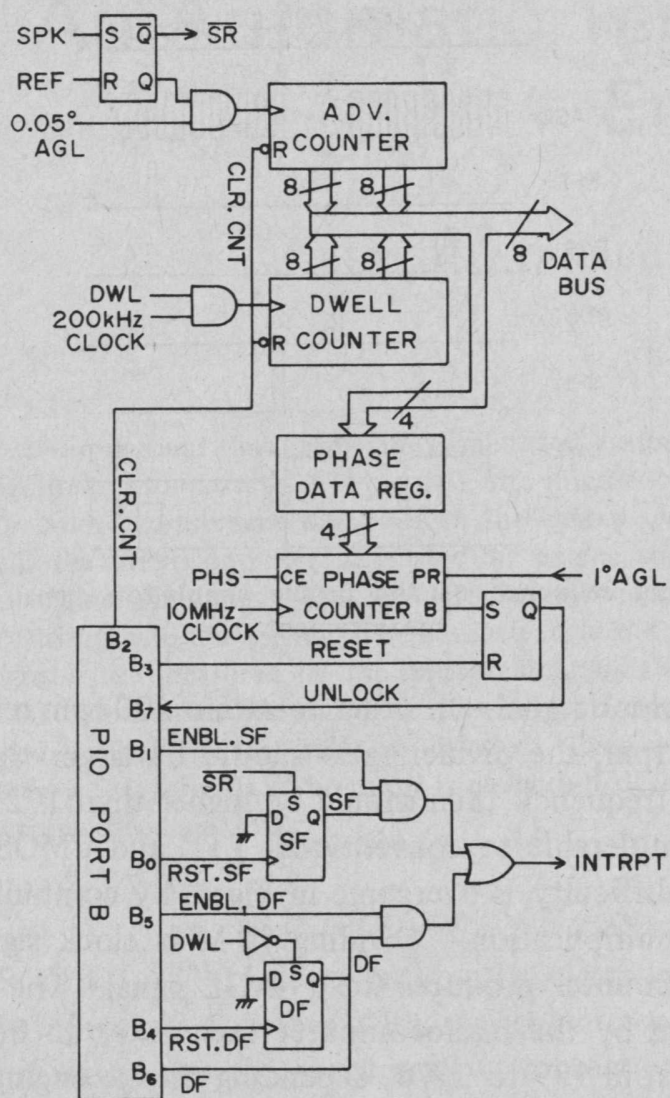

Fig. 4. The schematic diagram of the measurement unit.

operation of the engine simulator and then to generate the interrupt signal when the measurement is completed. The measurement timing is shown in Fig. 3.

The RS flip-flop uppermost in Fig. 4 produces the SR signal which assumes 1 during the period between the SPK and REF signals. The ADV counter measures the advance angle, $\theta_{s}$ in Fig. 3 , by counting the $0.05^{\circ}$-AGL during the SR signal. The accuracy is thus $0.1^{\circ} \mathrm{CA}$. The negative edge of the SR signal then sets the Spark Flag (SF) to inform that the advance measurement is over. This method of setting the SF requires the SPK signal, thereby eliminating the ambiguity which would otherwise be caused by using the REF signal for the trigger.

The DWL counter measures the dwell time $T_{D}$ in Fig. 3 by counting $200-\mathrm{kHz}$ clock signal during $T_{D}$. The count in the DWL counter is divided, after being stored in the microcomputer, by 10 using the software counter. The accuracy in the dwell measurement is thus $0.1 \mathrm{~ms}$. The negative edge of the DWL signal sets the Dwell Flag (DF) to inform that the dwell data are ready in the counter. Since only one port is available for the interrupt, the DF and SF are oRed to produce the interrupt signal. Upon acknowledging the interrupt signal, the microcomputer senses the $B_{6}$ line of the PIO port $B$ to distinguish between the DF and SF interrupts.

The phase data register and the phase counter form the phase comparator. The pulse width-modulated PHS signal is compared with the phase threshold stored in the phase data register. If its width, thus the phase difference between the $1^{\circ}$-AGL signals applied to the PLL, exceeds the threshold, the comparator produces the UNLOCK signal, informing the microcomputer that the operation of the engine simulator is

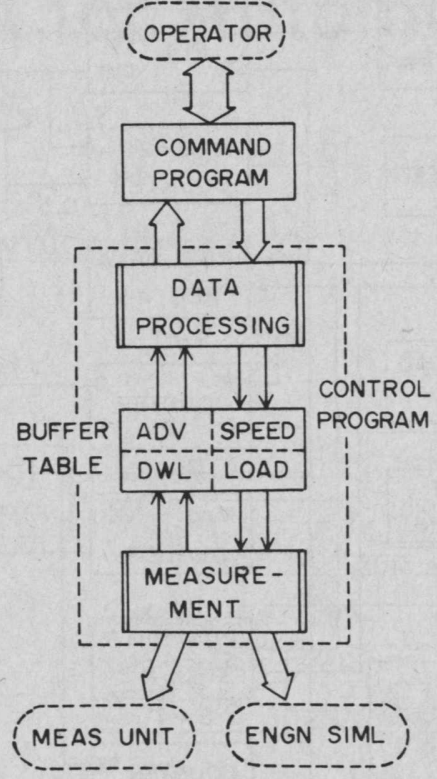

Fig. 5. The software structure of the ATE.

unstable. The measured advance and dwell data are then neglected. Otherwise, the microcomputer stores them into the buffer table and then sends the acknowledgment signals to reset all the counters and flags.

\section{B. Software Architecture}

The software structure of the ATE is shown in Fig. 5. It consists of the command program written in Basic and the control program in assembly language.

1) Command Program: The ATE communicates with an operator using this program. It asks an operator the following ignitor specifications:

1) positions of crankshaft position sensors, $\theta_{1}$ and $\theta_{2}$ in Fig. 3, references to TDC-the permissible range is from 3 to $255^{\circ}$ before TDC,

2) the lowest engine speed-the range between 600 and $10000 \mathrm{rpm}$ is permissible,

3) the highest engine speed-the range between 1000 and $12000 \mathrm{rpm}$ is permissible,

4) the speed resolution-the minimum resolution is 100 rpm,

5) the minimum and maximum engine load,

6) the engine load specified in this run-the load number that can be specified is 10 .

When the above questions are answered, the command program calls the control program using the instruction

$$
\text { CALL \#15, S, L, A, D. }
$$

Here, the arguments $\mathrm{S}$ and $\mathrm{L}$ are one-dimensional arrays in which the engine speed and load are stored, respectively. The arguments $\mathrm{A}$ and $\mathrm{D}$ are two-dimensional arrays in which the measured advance and dwell data are stored, respectively. The operation of the ATE is then managed by the control program.

2) Control Program: The flowchart of the control program is shown in Fig. 6. It includes the data processing and measurement subroutines. The frequency of advance and dwell data is so low that the buffer is provided for synchronizing 


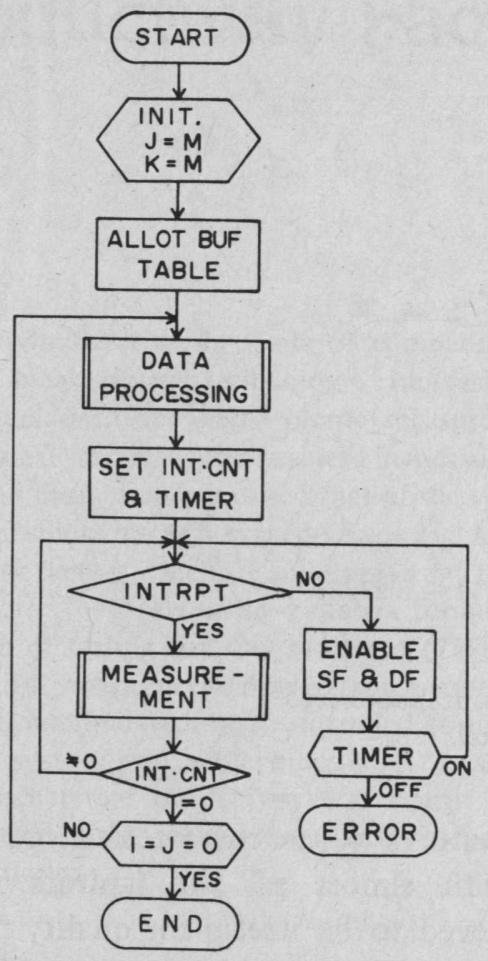

Fig. 6. The flowchart of the control program.
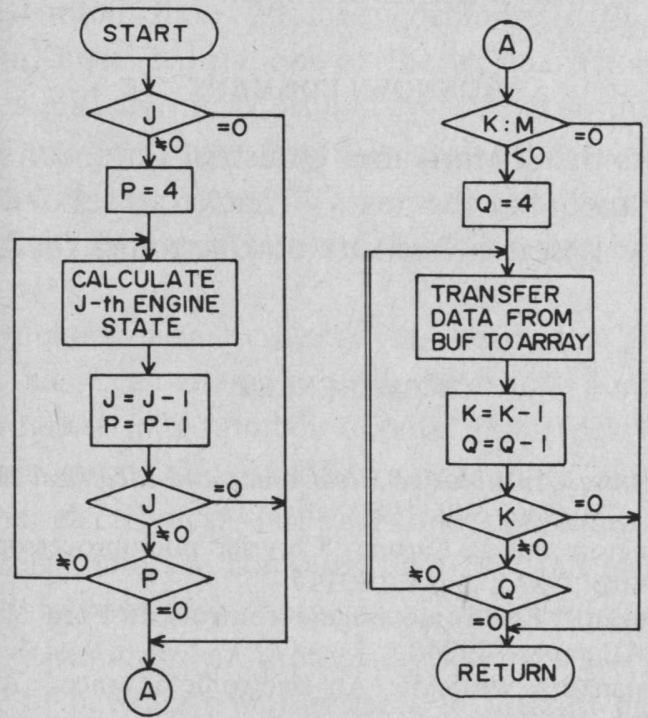

Fig. 7. The flowchart of the data processing subroutine.

between the two subroutines and thereby for increasing the throughput of the microcomputer. It is composed of four blocks labeled S, L, A, and D, and arranged in tabular form, as shown in Fig. 5. Each block has four registers. S and L blocks are used for storing the engine speed and load calculated in the data processing subroutine, and A and D blocks are used for storing the advance and dwell data obtained by he measurement subroutine, respectively.

First, the main program calculattes the total number of measurements based on the ignitor specifications and sets it into pointers $\mathbf{J}$ and $\mathrm{K}$ which indicate the engine state number and the measured data number, respectively. After assigning one section of memory area to the buffer table, it calls the data processing subroutine. This subroutine calculates four uccessive engine states and puts them into the buffer in order, is shown in the flowchart of Fig. 7. Here, the pointer $\mathrm{P}$ ad-

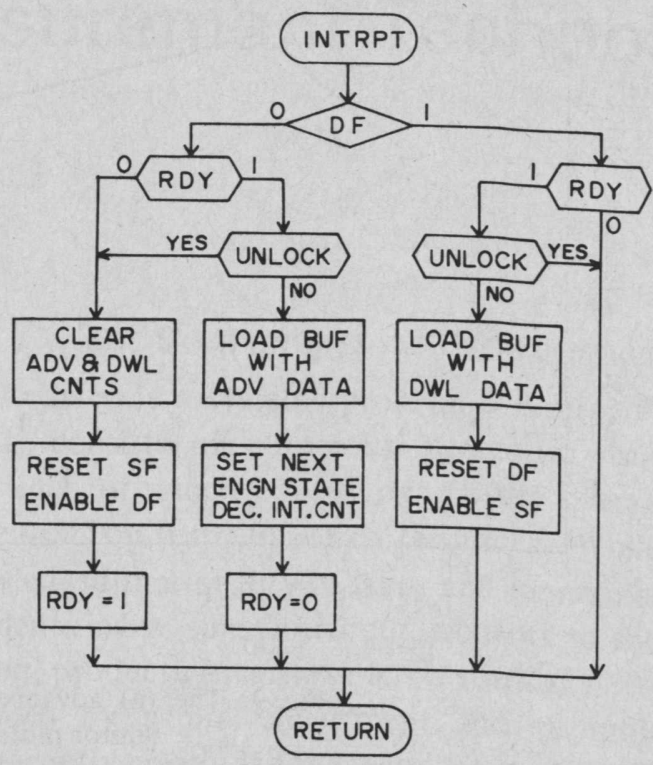

Fig. 8. The flowchart of the measurement subroutine.

dresses the paired speed and load register in the $\mathrm{S}$ and $\mathrm{L}$ blocks, while the pointer $\mathrm{Q}$ addresses the paired advance and dwell register in the A and D blocks. It also transfers four paired advance and dwell data successively from the buffer to the A and D arrays, if the data are ready in the buffer. After the data transfer, the management is returned again to the main program. Setting the interrupt counter (INT. CNT) and the software timer, it waits for the interrupt signal. If the interrupt signal does not appear within the interval set in the timer, the main program displays the error message "No spark signal is available" on a CRT and terminates the operation. Upon acknowledging the interrupt signal, on the other hand, it calls the measurement subroutine.

The flowchart of the measurement subroutine is shown in Fig. 8. It first distinguishes the DF from the SF. If the interrupt is caused by the DF, then it loads the D buffer with the data stored in the DWL counter after confirming the stable operation of the engine simulator. If the interrupt is caused by the SF, on the other hand, then it pushes the data stored in the ADV counter into the A buffer, sets the next engine state into the engine simulator, and resets the software switch RDY for steering the program flow. The DWL and ADV counters are reset by the following SF interrupt signal. This process is repeated until the INT.CNT becomes zero.

The main program repeats the data processing and measurement subroutines until all the engine states are covered. Then, the management is returned to the command program. The job remained is to display the result on a CRT, an $X-Y$ plotter, or a line printer, according to the output command.

\section{Test Examples}

Several ignitors now in use have been tested by the present ATE. Two results are given here to demonstrate its capability. Fig. 9(a) and (b) shows the advance angle and the dwell time, respectively, of a transistor ignitor measured under the maximum engine load condition. Designed to ignite four engines alternately, it has required four crankshaft position signals placed at $5,45,185$, and $225^{\circ}$ before TDC to control the advance and the dwell time. Fig. 10 shows the advance angle of 


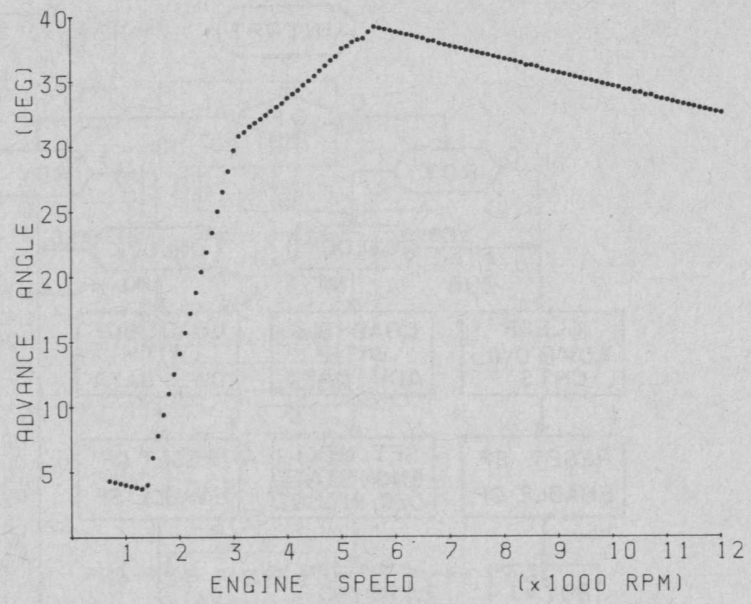

(a)

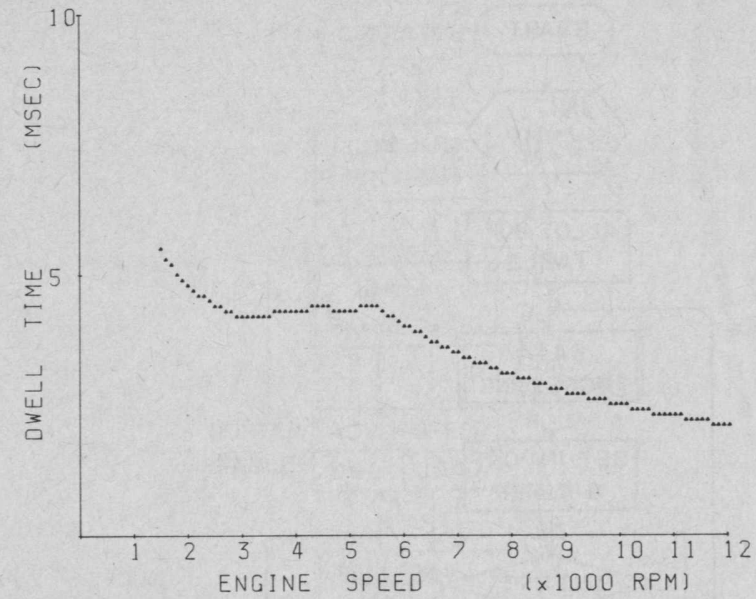

(b)

Fig. 9. The (a) advance angle and (b) dwell time of a transistorized ignitor measured as a function of engine speed.

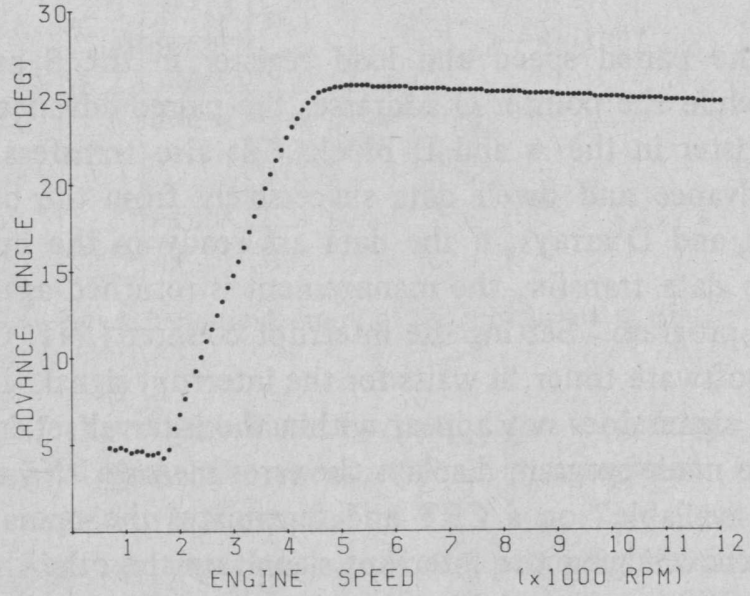

Fig. 10. The advance angle of a capacitor discharge ignitor measured as a function of engine speed.

the capacitor discharge ignitor (CDI) installed in a motorcycle. In this CDI, only two crankshaft position signals positioned at $5^{\circ}$ to $65^{\circ}$ before TDC have been required for the advance control. In either case, the measurement has been made every $100 \mathrm{rpm}$ from 700 to $12000 \mathrm{rpm}$. Time required for the test was only a few seconds. The accuracy of $0.1^{\circ}$-CA in the advance measurement and of $0.1 \mathrm{~ms}$ in the dwell-time measurement has been confirmed experimentally by comparing these results with those obtained by the hardware-oriented equipment described in [7].

\section{CONCLUSIONS}

An ATE for automotive engine ignitors has been described. Test examples have been also presented to demonstrate its capability. It features quick characterization, low cost, and compatibility with almost all the ignitors now available. Hence, it is believed to be useful for quality management in the production line. The extension to the ATE which allows characterization of not only ignitors but also closed-loop engine control systems is the future work.

\section{ACKNOWLEDGMENT}

The authors thank Moriyama Industrial Corp. for supplying the ignitors used for the test. Thanks are also due to $\mathrm{K}$. Kondo of the Research Institute of Electronics for his help in the test.

\section{REFERENCES}

[1] W. H. Crouse, Automotive Electronics and Electrical Equipment. New York: McGraw-Hill, 1981, chap. 16.

[2] J. Lappington and L. Caron, "Chrysler microprocessor spark advance control," SAE paper 780117.

[3] D. F. Hagen, "Electronic engine controls at Ford Motor Company," SAE paper 780842 .

[4] D. J. Simanaitis, "MISAR: An electronic advance," Auto. Eng., vol. 85 , Jan. 1977.

[5] C. M. Heinen and E. W. Beckman, "Balancing clean air against good mileage," IEEE Spectrum, vol. 14, pp. 47-50, Nov. 1977.

[6] LTX staff, "Automatic testing-Part 2; automotive electronics, Electron. Eng., vol. 55, pp. 63-68, Feb. 1983.

[7] K. Watanabe and A. Kitano, "Test instrument for electronic advance systems in automobiles," in Dig. Electrical and Electronic Measurement and Test Conf. (Ottawa, Ont., Canada), Sept. 1981, pp. 161-164.

[8] M Tümer and K. Watanabe, "A microprocessor-based diagnosis system for electronic ignitors," Bull. Res. Inst. Electro., Shizuoka Univer., vol. 16, pp. 135-141, Feb. 1982. 